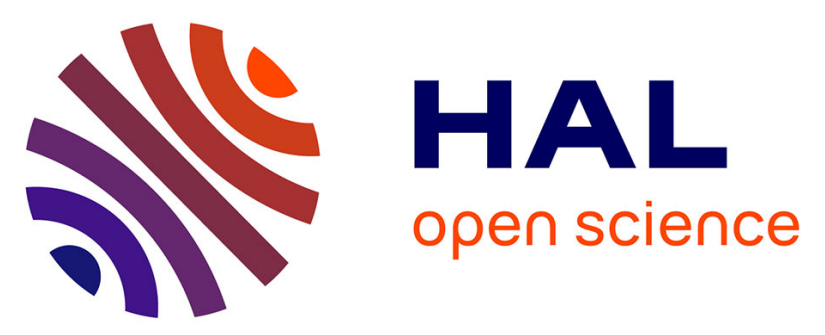

\title{
Charge distribution as a tool to investigate structural details. II. Extension to hydrogen bonds, distorted and hetero-ligand polyhedra
}

\author{
Massimo Nespolo, Giovanni Ferraris, Gabriella . Ivaldi, Rudolf Hoppe
}

\section{To cite this version:}

Massimo Nespolo, Giovanni Ferraris, Gabriella . Ivaldi, Rudolf Hoppe. Charge distribution as a tool to investigate structural details. II. Extension to hydrogen bonds, distorted and hetero-ligand polyhedra. Acta Crystallographica Section B : Structural Science, Crystal Engineering and Materials [2014-..], 2001, B57, pp.652-664. 10.1107/S0108768101009879 . hal-00130631

\section{HAL Id: hal-00130631 \\ https://hal.science/hal-00130631}

Submitted on 13 Feb 2007

HAL is a multi-disciplinary open access archive for the deposit and dissemination of scientific research documents, whether they are published or not. The documents may come from teaching and research institutions in France or abroad, or from public or private research centers.
L'archive ouverte pluridisciplinaire HAL, est destinée au dépôt et à la diffusion de documents scientifiques de niveau recherche, publiés ou non, émanant des établissements d'enseignement et de recherche français ou étrangers, des laboratoires publics ou privés. 


\section{Charge distribution as a tool to investigate structural details. II. Extension to hydrogen bonds, distorted and hetero-ligand polyhedra Massimo Nespolo, Giovanni Ferraris, Gabriella Ivaldi and Rudolf Hoppe}


Acta Crystallographica Section B

Structural

Science

ISSN 0108-7681

\section{Massimo Nespolo, ${ }^{\mathrm{a} *}$ Giovanni Ferraris, ${ }^{b}$ Gabriella Ivaldi ${ }^{b}$ and Rudolf Hoppe ${ }^{\mathrm{c}}$}

aJapan Science and Technology Corporation, National Institute for Materials Science, Advanced Materials Laboratory, 1-1 Namiki, Tsukuba-shi, Ibaraki 305-0044, Japan,

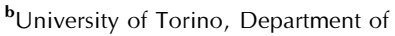
Mineralogical and Petrologic Sciences, Via Valperga Caluso 35, 10125 Torino, Italy, and cJustus-Liebig Universität, Institut für Anorganische und Analitische Chemie, Heinrich-BuffRing 58, D-35392 Giessen, Germany

Correspondence e-mail:

massimo.nespolo@nims.go.jp

\section{Charge distribution as a tool to investigate struc- tural details. II. Extension to hydrogen bonds, distorted and hetero-ligand polyhedra}

It is shown that one of the main reasons for most failures of the methods for calculating distance-dependent bond strengths is related to the distortion of the coordination polyhedra. The charge distribution (CD) method which depends on only one universal empirical parameter (contraction parameter) is modified to include: (i) an iterative calculation of the effective coordination number (ECoN), to deal with structures containing very distorted coordination polyhedra; (ii) a specific contraction parameter to treat structures containing any type of hydrogen bond; (iii) scale factors for coordination subshells, to treat structures with hetero-ligand polyhedra. The contraction parameter for the hydrogen bonds was obtained from 119 well refined structures based on neutron diffraction data. Examples of the application of the iterative charge distribution (CD-IT) are presented to show the efficiency of the new method in dealing with distorted (including hydrogen bonding) and hetero-ligand polyhedra. In particular, analysis of a series of 74 structures with pentacoordinated cations shows that deviations from overall trends are related to structure instability. The possible failure of the method with polyionic structures and 'dynamic' structures is discussed.

\section{Introduction}

The structure of inorganic crystals is commonly described in terms of concepts that, like oxidation numbers, coordination numbers and coordination polyhedra, have their roots in the XIX century theory of valence. Despite the approximations they implicitly contain, these concepts still represent today important tools to describe and elucidate crystal structures, provided a suitable extension is given. In both ionic and covalent compounds, we can assign positive and negative 'charges' (formal oxidation numbers) to each atom. For purely ionic bonds, these formal 'charges' closely represent the actual electrostatic charge of the ions. For covalent compounds, the formal 'charges' are rather a simplified way of representing the displacement of the electron density with respect to the isolated atoms. In both cases, the formal 'charges' are hereafter used to indicate the atoms (formally termed 'cations' and 'anions' without, however, any specific reference to the ionic bond) among which a bond may exist. For coordination polyhedra corresponding to the geometrically regular and semi-regular polyhedra all the cation-anion bonds have the same length and the coordination number is uniquely defined. In this case all the bonds around a cation have the same strength, which was defined as the ratio of the charge (formal oxidation number) of the cation and its coordination number (Pauling, 1929).
Received 17 May 2001

Accepted 14 June 2001
(C) 2001 International Union of Crystallography Printed in Great Britain - all rights reserved 
When the bonds around a cation span a certain range of lengths, Pauling's definition does not convey satisfactory information about the strength of the bonds. Besides, in those cases in which no net separation appears between a first and a second coordination sphere, the coordination number is hardly identified (Chiari, 1990; Balić Žunić \& Makovicky, 1996; Makovicky \& Balić Žunić, 1998). Also, the ionic radius is far from being uniquely defined, because the interatomic distances are not always describable as the sum of the ionic radii (Hoppe, 1970a, 1979), the variation depending partly on geometrical constraints on the crystal structure and especially on the overall connectivity of the atoms in the structure (Rutherford, 1991). Pauling's definition of bond strength has thus been extended by several authors, who introduced a dependence of the strength of a bond from its length. The common feature of these methods is the use of an empirical curve (' $R-s$ curve'), whose parameters are obtained by fitting on a large set of structures (see e.g. Brown \& Shannon, 1973; Brown \& Wu, 1976; Brown \& Altermatt, 1985). Given the experimental bond lengths and the paramters for a cation-anion pair, the bond strength is obtained from one of the $R-s$ curves and is termed 'bond valence' (BV; for a recent review see Urusov, 1995). Several extensions have been proposed with different goals, such as:

(i) to treat bonds which occur with a wide range of lengths (Brown, 1987);

(ii) to introduce the effect of the lone pairs on the geometry of the coordination polyhedra (Wang \& Liebau, 1996a,b);

(iii) to take into account zero and negative oxidation states (Naskar et al., 1997);

(iv) to correlate bond strength and electron density distribution (Gibbs et al., 1998);

(v) to treat compounds with hetero-ligand polyhedra (Mohri, 2000).

All these extensions are based on a more or less complex power-law relationship between the bond legth and the bond valence and do not have any internal criterion to evaluate whether the method itself is applicable or not to a given structure. Therefore, when a significant deviation from the expected bond valences occurs, the investigator should evaluate independently, and before applying the BV analysis, whether the quality of the refinement of the strucutre model is reliable and whether the type of structure (type and pattern of bonding) is suitable for the BV analysis. A 'blind' application without critical inspection may otherwise result in a discussion in terms of bond valences without solid ground.

Extensions in a different direction were proposed in terms of graph-theoretical approaches. Boisen et al. (1988) considered all the Lewis structures that can be associated with a given compound and defined the resonance bond number (RBN) as the weighted average of the bond order over the Lewis structures. This method ignores the periodicity of the crystalline structures and omits any non-nearest-neighbour interaction: the calculation requires $10^{7}$ or $10^{8}$ Lewis graphs per atom and the resonance bond numbers obtained in this way are close or identical to the bond valences, but the method has the advantage of being applicable to glasses as to crystalline solids (Rutherford, 1991, 1998).

A basically different approach is represented by the charge distribution (CD) method (Hoppe et al., 1989), which analyses the geometry of the coordination polyhedra through the effective coordination number, ECoN, uniquely defined in terms of the experimental bond lengths (Hoppe, 1979). Besides, the $\mathrm{CD}$ method possesses an internal criterion to evaluate the applicability, which consists of the $q / Q$ ratio for the cations (Nespolo et al., 1999). $q$ is the formal oxidation number and $Q$ the corresponding value computed as the weighted sum of the bond strength (termed 'bond weight' in the $\mathrm{CD}$ method) around the cations. When $(q / Q)_{\text {cations }}$ significantly diverges from 1 , either the refined structural model should be questioned or the structure in principle falls outside the limits of applicability of the method itself.

Inorganic compounds can be classified (Parthé, 1996) as normal valence compounds, where both cations and anions transfer all their valence electrons, and general valence compounds, where either the cations do not transfer all their valence electrons or the anions do not need as many electrons from the cations to complete their octet shells. General valence compounds are then subdivided into polycations (presence of cation-cation bonds or lone pairs) and polyanions (presence of anion-anion bonds). The geometrical definition of ECoN does not depend on the nature of the chemical bond and relies only on the pattern of bonding corresponding to the normal valence compound, but does not exclude compounds with lone pairs. The term polyion is used hereafter to include both polycations and polyanions, i.e. to indicate the presence of bonds between cations or between anions. The CD method (as well as other similar methods) in general is not applicable to polyions (see, however, the examples in §5.5), as well as compounds with delocalized electrons (metallic bond).

Non-polyionic structures which, despite the absence of errors in the refinement, could not be treated by the $C D$ method in its original formulation are typically structures with highly distorted coordination polyhedra, or with peculiar bonds like the hydrogen bond. In this paper we present an extension of the CD method which is also able to treat these types of structures.

\section{2. ${ }^{n} \mathrm{ECON}$}

The kernel of the method consists of a geometrical analysis of the coordination polyhedra and exploits ECoN, the effective coordination number (Hoppe, 1979). Hereinafter, the original equations have been rearranged and in some cases, for the purpose of generalization, a slightly different notation has been introduced.

Cation and anion sites are indicated as $M(i j)$ and $A(r s)$, respectively, where $i$ and $r$ identify the atomic species, $N_{i}^{+}$and $N_{r}^{-}$being the number of sites occupied by cations and anions of the same species, respectively, whereas $j$ and $s$ refer to the crystallographic type. For example, the mineral zunyite, $\mathrm{Al}_{12}(\mathrm{OH})_{18}\left(\mathrm{AlO}_{4}\right)\left(\mathrm{Si}_{5} \mathrm{O}_{16}\right) \mathrm{Cl}$ (Bartl, 1970), space-group type 
$F \overline{4} 3 m$, contains two crystallographically independent types of $\mathrm{Al}, \mathrm{Si}$ and $\mathrm{H}$, five types of $\mathrm{O}$ and one type of $\mathrm{Cl}$. In symbols it is thus represented as: $N_{1}^{+}=2, N_{2}^{+}=2, N_{3}^{+}=2, N_{1}^{-}=5, N_{2}^{-}=1$; $M(11) \sim M(12)=\mathrm{Al}, M(21) \sim M(22)=\mathrm{Si}, M(31) \sim M(32)=\mathrm{H}$, $A(11) \sim A(15)=\mathrm{O}, A(21)=\mathrm{Cl}$. The formal charges are indicated as $q(i j)$ and $q(r s) . q$ depends on $j$ or $s$ through the site occupation factor (s.o.f.). For s.o.f. $=1$, all the cations with the same $i$ and all the anions with the same $r$ have the same $q$. Similarly, the multiplicity of the Wyckoff position is indicated as $h(i j)$ and $h(r s)$. The $L$ th bond length between $M(i j)$ and $A(r s)$ is indicated as $d(i j \rightarrow r s)_{L}$. For computational purposes, the bond lengths are classified in increasing length with respect to $i, j$ and $r, d(i j \rightarrow r s)_{1}$ being the shortest and $s$ a sort of dummy index at this stage.

Pauling (1929) pointed out that for ionic compounds the distance between two ions with charges of opposite sign, among which an ionic bond exists, should be nearly identical to the sum of the ionic radii $R(i)$ and $R(r)$. Hoppe (1979) defined the fictive ionic radii, $\operatorname{FIR}(i j \rightarrow r s)_{L}$, as

$$
\operatorname{FIR}(i j \rightarrow r s)_{L}=d(i j \rightarrow r s)_{L} \cdot \frac{R(i)}{R(i)+R(r)} .
$$

Excluding special features such as double bonds, $\operatorname{FIR}(i j \rightarrow r s)_{1}$ should correspond to $R(i)$, whereas $\operatorname{FIR}(i j \rightarrow r s)_{L>1}$ increases with $L$. If $m(i j \rightarrow r s)_{L}$ is the multiplicity of the bond $d(i j \rightarrow$ $r s)_{L}$, the mean fictive ionic radius, ${ }^{n}$ MEFIR, is calculated through an iterative process

$$
\begin{aligned}
{ }^{n} \operatorname{MEFIR}(i j \rightarrow r)= & \left\{\sum_{s} \sum_{L} \operatorname{FIR}(i j \rightarrow r s)_{L} \cdot m(i j \rightarrow r s)_{L}\right. \\
& \left.\cdot \exp \left\{1-\left[\frac{\operatorname{FIR}(i j \rightarrow r s)_{L}}{{ }^{n-1} \operatorname{MEFIR}(i j \rightarrow r)}\right]^{6}\right\}\right\} \\
& \times\left\{\sum_{s} \sum_{L} m(i j \rightarrow r s)_{L}\right. \\
& \left.\cdot \exp \left\{1-\left[\frac{\operatorname{FIR}(i j \rightarrow r s)_{L}}{{ }^{n}-1} \operatorname{MEFIR}(i j \rightarrow r)\right]\right\}\right\}^{-1} \\
= & \frac{R(i)}{R(i)+R(r)} \\
& \cdot\left\{\sum_{s} \sum_{L} d(i j \rightarrow r s)_{L} m(i j \rightarrow r s)_{L}\right. \\
& \left.\cdot \exp \left\{1-\left[\frac{d(i j \rightarrow r s)_{L}}{n-1}\right]^{6}\right\}\right\} \\
& \times\left\{\sum_{s} \sum_{L} m(i j \rightarrow r s)_{L}\right. \\
& \left.\cdot \exp \left\{1-\left[\frac{d(i j \rightarrow r s)_{L}}{n-1} d(i j \rightarrow r)\right]^{6}\right\}\right\}^{-1},
\end{aligned}
$$

where $n$ is the number of iterations. The following equalities hold

$$
\begin{aligned}
& { }^{0} \operatorname{MEFIR}(i j \rightarrow r)=\mathrm{FIR}(i j \rightarrow r s)_{1} \\
& { }^{0} d(i j \rightarrow r)=d(i j \rightarrow r s)_{1} .
\end{aligned}
$$

The weighted mean distance ${ }^{n} d(i j \rightarrow r)$ is (cf. Hoppe et al., 1989)

$$
\begin{aligned}
{ }^{n} d(i j \rightarrow r)= & \left\{\sum_{s} \sum_{L} d(i j \rightarrow r s)_{L} m(i j \rightarrow r s)_{L}\right. \\
& \left.\cdot \exp \left\{1-\left[\frac{d(i j \rightarrow r s)_{L}}{n-1}\right]^{6}\right\}\right\} \\
& \times\left\{\sum_{s} \sum_{L} m(i j \rightarrow r s)_{L}\right. \\
& \left.\left.\cdot \exp \left\{1-\left[\frac{d(i j \rightarrow r s)_{L}}{n-1}\right]^{6}\right\}\right\}^{-1}(i j \rightarrow r)\right] \\
= & \frac{R(i)+R(r)}{R(i)} \cdot{ }^{n} \operatorname{MEFIR}(i j \rightarrow r) .
\end{aligned}
$$

The definitions introduced in (3) permit the unification into a single equation, (4), of the two equations (2) and (5) given in Hoppe (1979).

The 'strength' of the $d(i j \rightarrow r s)_{L}$ bond is measured by the bond weight ${ }^{n} \mathrm{BW}(i j \rightarrow r s)_{L}$, as defined by Nespolo et al. (1999)

$$
\begin{aligned}
{ }^{n} \mathrm{BW}(i j \rightarrow r s)_{L} & =\exp \left\{1-\left[\frac{d(i j \rightarrow r s)_{L}}{{ }^{n} d(i j \rightarrow r)}\right]^{6}\right\} \\
& =\exp \left\{1-\left[\frac{\operatorname{FIR}(i j \rightarrow r s)_{L}}{{ }^{n} \operatorname{MEFIR}(i j \rightarrow r)}\right]^{6}\right\}
\end{aligned}
$$

and the effective coordination number ${ }^{n} \mathrm{ECoN}$ is defined both in terms of $M(i j)$ and $A(r),{ }^{n} \mathrm{ECoN}(i j \rightarrow r)$ and of $M(i j)$ alone, ${ }^{n} \mathrm{ECoN}(i j)$

$$
\begin{aligned}
{ }^{n} \mathrm{ECoN}(i j \rightarrow r) & =\sum_{s} \sum_{L} m(i j \rightarrow r s)_{L} \cdot{ }^{n} \mathrm{BW}(i j \rightarrow r s)_{L} \\
{ }^{n} \mathrm{ECoN}(i j) & =\sum_{r}^{n} \mathrm{ECoN}(i j \rightarrow r) .
\end{aligned}
$$

Mohri (2000) defined 'homo-ligand polyhedra' and 'heteroligand polyhedra' coordination polyhedra as made by only one $(r=1)$ or more than one $(r>1)$ type of anion, respectively. For homo-ligand polyhedra ${ }^{n} \mathrm{ECoN}(i j \rightarrow r)$ and ${ }^{n} \mathrm{ECoN}(i j)$ coincide. Instead, for hetero-ligand polyhedra, ${ }^{n} \mathrm{ECoN}(i j)$ defined simply as the sum of ${ }^{n} \mathrm{ECoN}(i j \rightarrow r)$ treats independently the coordination shells made by each type of anion, a problem which is discussed in the next section.

Equations (5) and (6) show that ECoN is equivalently defined in terms of MEFIR (as in Hoppe, 1979) and of $d(i j \rightarrow$ $r$ ) (as in Hoppe et al., 1989). MEFIR is the only variable that depends, through FIR, on the ionic radii. BW, ECoN and the computed 'charges', defined and illustrated in the next section (which depend on ECoN), do not depend on the ionic radii.

$\operatorname{MEFIR}(i j \rightarrow r)$ and $d(i j \rightarrow r)$ are computed in an iterative way: the result of the computation at the $(n-1)$ th stage is used as an input value for the $n$th stage, and the computation terminates when the difference between ${ }^{n-1} \operatorname{MEFIR}(i j \rightarrow r)$ and ${ }^{n} \operatorname{MEFIR}(i j \rightarrow r)$, or between ${ }^{n-1} d(i j \rightarrow r)$ and ${ }^{n} d(i j \rightarrow r)$, is negligible (for practical purposes, set to 0.001 ).

$\mathrm{ECoN}$ is in general a non-integer number giving a weighted measure of the coordination environment of a cation site. For regular or semi-regular coordination polyhedra the compu- 
tation terminates with ${ }^{1} \operatorname{MEFIR}(i j \rightarrow r)$ and ${ }^{1} d(i j \rightarrow r)$, and ${ }^{1} \mathrm{ECoN}$ reduces to the classical integer value corresponding to the number of $A(r s)$ coordinated by $M(i j)$. Instead, the larger the distortion of a polyhedron (deviation from its ideal shape), the larger the number of iterations and the larger the deviation of ${ }^{n} \mathrm{ECoN}$ from the classical coordination number.

In the previous version of the CD method (Hoppe et al., 1989; Nespolo et al., 1999), no iterations were employed, and the charge distribution was computed in terms of ${ }^{1} \mathrm{ECoN}$ : as a consequence, the method was limited in its applications to structures with coordination polyhedra of relatively limited distortion. The use of ${ }^{n} \mathrm{ECoN}$ instead permits to treat structures also containing distorted polyhedra.

\section{Iterative charge distribution}

The clue of the CD method is actually the distribution of ${ }^{n} \mathrm{ECoN}(i j \rightarrow r)$ among all the bonds around $M(i j)$

$$
\Delta^{n} \mathrm{ECoN}(i j \rightarrow r s)=\frac{\sum_{L} m(i j \rightarrow r s)_{L}{ }^{n} \mathrm{BW}(i j \rightarrow r s)_{L}}{{ }^{n} \mathrm{ECoN}(i j \rightarrow r)} .
$$

$\Delta^{n} \mathrm{ECoN}$ gives the contribution by the $A(r s)$ anion to ${ }^{n} \mathrm{ECoN}(i j \rightarrow r)$. The fraction of the formal charge $q(i j)$ that the cation $M(i j)$ shares with the anion $A(r s)$ is obtained by simply multiplying $\Delta^{n} \mathrm{ECoN}$ by $q(i j)$ and by introducing a scale factor ${ }^{n^{\prime}} F(i j \rightarrow r)$ for hetero-ligand polyhedra

$$
\Delta^{n^{\prime}} q(i j \rightarrow r s)=\Delta^{n} \mathrm{ECoN}(i j \rightarrow r s) q(i j)^{n^{\prime}} F(i j \rightarrow r) .
$$

The computed 'charge' of the anions is then obtained by summing up each fraction of charge, taking into account, however, the ratio of the multiplicities of the respective Wyckoff positions (to avoid counting more than once the contributions from the same cation)

$$
{ }^{n^{\prime}} Q(r s)=-\sum_{i} \sum_{j} \Delta^{n^{\prime}} q(i j \rightarrow r s) \frac{h(i j)}{h(r s)} .
$$

The 'charge' of the cation $M(i j)$ is computed as the weighted sum of $q(r s) /^{\prime \prime} Q(r s)$ for the anions $A(r s)$ bonded to $M(i j)$, where the weight is the fraction of shared charge $q(i j)$ defined by (8)

$$
\begin{aligned}
Q(i j)= & \sum_{r} \sum_{s} \Delta^{n^{\prime}} q(i j \rightarrow r s) \frac{q(r s)}{n^{\prime} Q(r s)} \\
= & {\left[\sum_{r} \sum_{s} \Delta^{n} \mathrm{ECoN}(i j \rightarrow r s) \frac{q(r s)}{n^{\prime} Q(r s)} n^{n^{\prime}} F(i j \rightarrow r)\right] } \\
& \times q(i j) .
\end{aligned}
$$

A structure which is correctly solved and perfectly valencebalanced has both $q(r s) /^{n^{\prime}} Q(r s)$ and $q(i j) / Q(i j)$ ideally equal to 1. As shown below, structural strains affect $q(r s) / n^{\prime} Q(r s)$, which deviates from 1: in this case we speak of the over-underbonding (OUB) effect.

For homo-ligand polyhedra, $n^{\prime}=1$ (no iterations) and ${ }^{n^{\prime}} F(i j \rightarrow r)={ }^{1} F(i j \rightarrow 1)=1:(8)$ thus reduces to $(5 a)$ in Hoppe et al. (1989), with the only difference that we consider being the ratio $h(i j) / h(r s)$ in (9), to emphasize the role of
$\Delta^{n^{\prime}} q(i j \rightarrow r s)$ in both $Q(r s)$ and $Q(i j)$. Instead, for heteroligand polyhedra the scale factors are computed through an iterative procedure that makes them independent from the definition of atomic or ionic radius, and is formally reminiscent of the iterative calculation of bond valences that Brown (1977) adopted to apply his 'equal valence rule'. The starting value is taken as

$$
{ }^{1} F(i j \rightarrow r)=1 / N(i j \rightarrow r)
$$

where $N(i j \rightarrow r)$ is the number of different types of anions [different $r$ in $A(r)$ ] coordinated by $M(i j)$. Equation (11) assumes that all the anions surrounding $M(i j)$ have the same radius, which corresponds to degeneration to the case of homo-ligand polyhedra, when $N(i j \rightarrow r)=1$ and ${ }^{1} F(i j \rightarrow r)=1$. For the general case of $N(i j \rightarrow r)>1$, (11) overestimates the contributions from the type of anions with larger radius and underestimates the contribution from smaller anions. This results in too negative ${ }^{1} Q(r s)$ for larger anions and too positive ${ }^{1} Q(r s)$ for smaller anions. This apparent OUB effect is accounted for by iterating the calculation of ${ }^{n^{\prime}} F(i j \rightarrow r)$ in the following way. If ${ }^{n^{\prime}-1} \delta Q(r)$ is the weighted difference between $Q(r)$ and $q(r)$ at the $\left(n^{\prime}-1\right)$ th iteration stage, and $\left|n^{\prime}-1 \delta Q(r)\right|$ is the largest value of ${ }^{n^{\prime}-1} \delta Q(r)$ among all the anions, ${ }^{n^{\prime}} F(i j \rightarrow$ $r$ ) is computed as

$$
\begin{aligned}
& { }^{n^{\prime}-1} \delta Q(r)=\frac{\sum_{s}\left[{ }^{n^{\prime}-1} Q(r s)-q(r s)\right] h(r s)}{\sum_{s} h(r s)} \\
& { }^{n^{\prime}-1} \Delta F=0.1 \cdot \log _{10}^{-1}\left\{\operatorname{int}\left[\log _{10}\left|\overline{\mid n^{\prime}-1} \delta Q(r)\right|\right]\right\}
\end{aligned}
$$

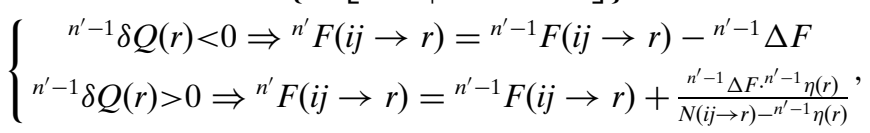

where the function 'int' reports the argument rounded up to the largest integer and ${ }^{n^{\prime}-1} \eta(r)$ is the number of anions for which $n^{\prime}-1 \delta Q(r)<0$, which in general varies during the iteration process. In practice, ${ }^{n^{\prime}}-1{ }^{1} \Delta F$ is a dumping factor always taken one digit lower than $\mid \frac{n^{\prime}-1}{\delta Q(r) \mid}$ : the logarithmic function simply reports 1 in the first non-zero digit of $\left|n^{\prime}-1 \delta Q(r)\right|$. The iteration in (12) continues until convergence, that for practical purposes is set as $\sum_{r}\left|n^{\prime} \delta Q(r)-{ }^{n^{\prime}-1} \delta Q(r)\right| \leq 0.001 .{ }^{n^{\prime}} F(i j \rightarrow r)$ is decreased or increased by $n^{\prime}-1 \Delta F$ for anions whose weighted $Q(r)$ at the $\left(n^{\prime}-1\right)$ th iteration stage is too negative [negative values of $\left.n^{\prime}-\delta Q(r)\right]$ or too positive [positive values of $n^{\prime}-1 \delta Q(r)$ ], respectively. The dependence of ${ }^{n^{\prime}} F(i j \rightarrow r)$ on $i j$ is inherited from the definition of ${ }^{1} F(i j \rightarrow r)=1 / N(i j \rightarrow r)$.

In (12) the scale factors for the different anions by definition always sum up to 1

$$
\sum_{r}^{n^{\prime}} F(i j \rightarrow r)=1
$$

This preserves the total negative charge and thus the electroneutrality of the formula unit, expressed as 


$$
\begin{aligned}
-\sum_{r} \sum_{s} q(r s) h(r s) & =\sum_{i} \sum_{j} q(i j) h(i j) \\
& =-\sum_{r} \sum_{s}^{n^{\prime}} Q(r s) h(r s) \\
& =\sum_{i} \sum_{j} Q(i j) h(i j) .
\end{aligned}
$$

A similar consideration is the basis of the algorithm to compute the scale factors. Equation (12) implicitly assumes that a real OUB effect affects mainly the anionic charges of each single anion, but without grossly modifying the total charge of each type of anion. A large deviation of $\sum_{s}{ }^{1} Q(r s) h(r s)$ from $\sum_{s} q(r s) h(r s)$ is thus considered mainly an apparent OUB effect, due to the different sizes of $A(r)$ to be accounted for by introducing the scale factor. However, $\sum_{s} n^{\prime} Q(r s) h(r s)$ is not forced to become identical with $\sum_{s} q(r s) h(r s)$, because the convergence is monitored by $\delta Q(r)$. The iteration on ${ }^{n^{\prime}} F(i j \rightarrow r)$ in fact terminates when $\delta Q(r)$ does not change in two successive cycles.

Two considerations are important to elucidate the meaning of (10) [cf. (7b) in Nespolo et al., 1999].

(i) $q(i j)$ does not play any essential role, all the information being conveyed by the factors within the parentheses, which sum up to 1 for $Q(i j)=q(i j)$. The introduction of $q(i j)$ is useful to maintain the same 'ionic' terminology in terms of 'charge' originally introduced by Pauling (1929).

(ii) $Q(i j)$ represents the distribution of $q(r s) /{ }^{n^{\prime}} Q(r s)$ among the cations and (10) without the factor $q(r s) /{ }^{n^{\prime}} Q(r s)$ would be just an identity $Q(i j)=q(i j)$.

In fact, remembering (6), (7) and (13)

$$
\begin{aligned}
& \sum_{r} \sum_{s} \Delta^{n} \mathrm{ECoN}(i j \rightarrow r s)^{n^{\prime}} F(i j \rightarrow r)= \\
& \sum_{r}^{n^{\prime}} F(i j \rightarrow r) \sum_{s} \Delta^{n} \mathrm{ECoN}(i j \rightarrow r s) \\
& =\sum_{r}^{n^{\prime}} F(i j \rightarrow r) \cdot 1=1 .
\end{aligned}
$$

When a real OUB effect is present, some $q(r s) /{ }^{n^{\prime}} Q(r s)$ ratios deviate from 1 , but all the $q(r s){ }^{n^{\prime}} Q(r s)$ ratios corresponding to $A(r s)$ bonded to a given cation enter in the calculation of $Q(i j)$ in (10) and $q(i j) / Q(i j)$ should not be affected by the OUB effect. Reasons for $q(i j) / Q(i j)$ significantly deviating from 1 can be:

(i) the refined structure model is inadequate including overlooked (light) atoms and disorder;

(ii) wrong assignment of oxidation numbers, including the case of sites with isomorphous substitutions;

(iii) presence of polyions, which are not accounted for by the $M(i j) \rightarrow A(r s)$ bonding patterns assumed by the CD method (see §5.5);

(iv) the coordination of one or more cations is so distorted that the polyhedral description is too approximate.

When instead $q(i j) / Q(i j)$ is reasonably close to $1,{ }^{n^{\prime}} Q(r s)$, ${ }^{n} \mathrm{ECoN}(i j \rightarrow r)$ and, with reference to each bond, ${ }^{n} \mathrm{BW}(i j \rightarrow r)_{L}$ are suitable parameters to investigate structural changes, e.g. as a function of composition, temperature and pressure (Nespolo et al., 1999; Nespolo, Sato et al., 2000). The only trivial exceptions are those of structures containing either only one independent cation site or only one independent anion site, for which $Q(i j)=q(i j)$ is always realised. In case of only one independent cation site, substituting (8) and (9) into (10) for $i=1$ and $j=1$

$$
Q(11)=\sum_{r} \sum_{s}-q(r s) \frac{h(r s)}{h(11)}=q(11),
$$

where the last equivalence originates from (14). Instead, in the case of only one anion site, from (6) and (7) with $r=s=1$ it turns out that $\Delta^{n} \mathrm{ECoN}(i j \rightarrow 11)=1$ and $F(i j \rightarrow 11)=1$ by definition. It follows that:

$$
Q(i j)=\frac{q(11)}{Q(11)} q(i j)=\frac{q(11) h(11)}{\sum_{i^{\prime}} \sum_{j^{\prime}} q\left(i^{\prime} j^{\prime}\right) h\left(i^{\prime} j^{\prime}\right)} q(i j)=q(i j),
$$

where the first equivalence comes from (10) with $\Delta^{n} \mathrm{ECoN}(i j$ $\rightarrow 11)=1$ and $F(i j \rightarrow 11)=1$, and the second equivalence comes from (14) with $r=1$ and $s=1$.

The application of the CD method to investigate cases of inadequate refinement was illustrated for the homologous series $\mathrm{LuFeO}_{3}(\mathrm{ZnO})_{n}$ (Nespolo, Nakamura \& Ohashi, 2000), and for the compounds $\mathrm{YbFeMnO}_{4}$ (Nespolo et al., 2000a) and $\mathrm{ErFeMnO}_{4}$ (Nespolo et al., 2000b). The deviation of $q(i j) / Q(i j)$ from 1 for the average structure prompted for a further refinement, which showed incomplete occupation $\left(\mathrm{YbFeMnO}_{4}\right)$ or statistical distribution $\left(\mathrm{ErFeMnO}_{4}\right.$ and $\mathrm{LuFeZnO}_{4}$ ) of the rare-earth cation. These conclusions are unaffected by the iteration procedure introduced here, because the convergence on ${ }^{n} d(i j \rightarrow r)$ in those examples is almost immediate and ${ }^{n} \mathrm{ECoN}$ is practically identical with ${ }^{1} \mathrm{ECoN}$.

\section{Charge distribution for structures including hydrogen bonds}

From the viewpoint of the coordination geometry, the hydrogen bond $A 1-\mathrm{H} \cdots A 2$ has some features that make it unique and not treatable by the $C D$ method in its original form:

(i) the $\mathrm{H}$ atom has only two bonds except in the rare cases of multiple hydrogen bonds;

(ii) very short $A 1-\mathrm{H}$ distances;

(iii) large relative gap in length between the donor and the acceptor bonds, $\delta 21=d(i j \rightarrow r s)_{2}-d(i j \rightarrow r s)_{1}$ (Chiari \& Ferraris, 1982).

These features result in a high ratio $d(i j \rightarrow r s)_{2} / d(i j \rightarrow r s)_{1}$ and in practice the bond with the acceptor, $d(i j \rightarrow r s)_{2}$, is overlooked by (2) and (4). The hydrogen bond is in this respect unique because other cations may show the same order of gap $\delta 21$, but the longer bond lengths result in a much lower $d(i j \rightarrow$ $r s)_{2} / d(i j \rightarrow r s)_{1}$ ratio and $d(i j \rightarrow r s)_{2}$ is thus accounted for by (2) and (4).

The only empirical parameter employed by the CD method is the so-called contraction parameter (Nespolo et al., 1999), which consists of the exponent 6 in (2) and (4). This contraction parameter was chosen because it resulted in ${ }^{1} \mathrm{ECoN}$, which is identical with the classical coordination number for simple and regular structures (Hoppe, 1979). However, the decreasing weight it induces with the increasing distances $d(i j \rightarrow r s)_{L}$ is too rapid for the case of hydrogen 
bonds. For $M(i j)=\mathrm{H}$ a different contraction parameter should be used, in order not to overlook the bond with the acceptor. To obtain a parameter specific for the hydrogen bond we have extracted from the Inorganic Crystal Structure Database (Bergerhoff et al., 1983) a large number of structures containing $\mathrm{H}$ atoms and refined by single-crystal neutron diffraction data. Among them we have selected a set of structures on the basis of the following criteria:

(i) the presence of different types of donors and acceptors;

(ii) more than one site for both cations and anions, otherwise the equivalence $Q(i j)=q(i j)$ is always verified, see (16) and (17);

(iii) elimination of identical structures appearing more than once in the database;

(iv) elimination of the structures with evident anomalies;

(v) elimination of the structures for which the ratio $q(i j) /$ $Q(i j)$ largely deviated from 1 for any contraction parameter we tried, thus suggesting the reason for the deviation was not in the treatment of the hydrogen bond.

In this way we have obtained a set of 119 structures, reported in Table 1, on which we have refined the contraction parameter. The overall deviation of $Q$ with respect to $q$ is measured by the parameter

$$
\sigma=\left\{\sum_{i} \sum_{j}[q(i j)-Q(i j)]^{2} / N-1\right\}^{1 / 2},
$$

which reduces to the classical standard uncertainty for atoms all with the same $q$. The contraction parameter for the hydrogen bond was fixed at the value that minimized the mean value of $\sigma$ taken on the entire set of structures both for the $\mathrm{H}$ atoms alone, $\left\langle\sigma_{\mathrm{H}}\right\rangle=\sum_{i=1}^{N}\left(\sigma_{H}\right)_{i} / N$, and for all the cations, $\left\langle\sigma_{\text {cat }}\right\rangle=\sum_{i=1}^{N}\left(\sigma_{\text {cat }}\right)_{i} / N$, where $N=119$. The contraction parameter found in this way is 1.6.

It should be taken into account that the large errors in the charge distribution of structures containing $\mathrm{H}$ atoms determined by X-ray diffraction may suggest that the positions of those atoms are too approximate. In these cases, as well as when the positions of the $\mathrm{H}$ atoms are unknown, curves of bond strength versus $\mathrm{O} \cdots \mathrm{O}$ (Ferraris \& Ivaldi, 1988) can be used to evaluate the bond strength of $\mathrm{O} \cdots \mathrm{O}$ hydrogen bonds. The coordinates of the $\mathrm{H}$ atoms can also be calculated on the basis of geometric and force-field considerations (Nardelli, 1999).

In those cases in which one or more hydrogen are present but do not form hydrogen bonds, being instead bonded to only one anion, it is not necessary to know their position. These $\mathrm{H}$ atoms can be simply ignored, provided the charge $q(r s)$ of the anions to which they are bonded is increased by one unit. For example, the case of an $\mathrm{O}-\mathrm{H}$ bond can be treated by assigning $q=-1$ to the $\mathrm{O}$ atom and neglecting the $\mathrm{H}$ atom. An example can be found in Ferraris et al. (2001).

\section{Examples}

Here we present some examples which demonstrate the improvements brought by the new approach with respect to the original, non-iterative $\mathrm{CD}$ method (further examples can be found in Nespolo et al., 2001). All the computations have been made with the CHARDI-IT program, which can be obtained on request from the first author or downloaded from the site http://takechan.kiso.tsukuba.ac.jp/ecasig5/CD.htm.

\subsection{Structures with distorted polyhedra}

Table 2 shows the results of the $\mathrm{CD}$ and CD-IT computation for $\mathrm{V}_{1.08} \mathrm{P}_{0.92} \mathrm{O}_{5}$, space-group type $P 4 / n$ (Jordan \& Calvo, 1976). The non-iterative CD gives not so satisfactory results, as shown by the large deviation of $q(i j)$ from $Q(i j)$. The trigonal bipyramids of oxygen anions around the $\mathrm{V}$ site are highly distorted, and results in ${ }^{1} \mathrm{ECoN}(i=1, j=1)=2.86$. The iterative $\mathrm{CD}$ gives ${ }^{10} \mathrm{ECoN}(i=1, j=1)=3.81$ and a much better distribution of the formal charges. The BV calculation (results according to the three most commonly employed curves are shown) is unable to treat distorted polyhedra like these, as shown by the large $q / Q$ ratios.

\subsection{Structures with hydrogen bonds}

The example of $\mathrm{NaH}_{2} \mathrm{PO}_{4} \cdot \mathrm{H}_{2} \mathrm{O}$, space-group type $P 2_{1} 2_{1} 2_{1}$ (Bartl et al., 1976), is shown in Table 3, where five of the six crystallographically independent $\mathrm{H}$ atoms $(\mathrm{H} 1-\mathrm{H} 3, \mathrm{H} 5, \mathrm{H} 6)$ are involved in hydrogen bonds. For $\mathrm{H} 4$, the distance from the acceptor, $d(\mathrm{H} 4 \cdots \mathrm{O} 4)=2.25 \AA$, indicates that if still present the hydrogen bond can be only very weak (Chiari \& Ferraris, 1982). In fact, zero contribution to ${ }^{n} \mathrm{ECoN}$ is obtained. The non-iterative $\mathrm{CD}$ method, with contraction parameter fixed at 6, gives ${ }^{1} \mathrm{ECoN}=1.00$ for all the $\mathrm{H}$ atoms, completely overlooking all the hydrogen bonds. The CD-IT method, with contraction parameter 1.6 for the $\mathrm{H}$ atoms, gives ${ }^{n} \mathrm{ECoN}$ from 1.43 to 1.85 for the five hydrogen atoms forming hydrogen bonds and satisfactory charge distribution for CD-IT. The BV calculation (results according to the same curves used in Table 2, both in their original formulation and modified for $\mathrm{H}-\mathrm{O}$ bonds according to Brown, 1987) is also unable to satisfactorily treat the hydrogen bond. A calculation with the program HYDROGEN (Nardelli, 1999) of the hydrogen coordinates and of the length of the hydrogen bond on the basis of the $Q(i j)$ in Table 3 shows systematic better agreement with the experimental data than using the charges obtained by simple stoichiometry (M. Nardelli, personal communication).

\subsection{Structures with hetero-ligand polyhedra}

The example of $\mathrm{La}_{2} \mathrm{SeSiO}_{4}$, space-group type $\mathrm{Pbcm}$ (Brennan \& Ibers, 1991), is shown in Table 4. The two La cations coordinate $3 \mathrm{Se}+6 \mathrm{O}$ and $4 \mathrm{Se}+6 \mathrm{O}$, respectively, whereas $\mathrm{Si}$ is in a slightly distorted tetrahedron of $\mathrm{O}$ atoms. The coordination polyhedra are described by ${ }^{5} \mathrm{ECoN}(\mathrm{La} 1 \rightarrow$ $\mathrm{Se})=2.6,{ }^{2} \mathrm{ECoN}(\mathrm{La} 1 \rightarrow \mathrm{O})=5.9,{ }^{6} \mathrm{ECoN}(\mathrm{La} 2 \rightarrow \mathrm{Se})=3.1$, ${ }^{2} \mathrm{ECoN}(\mathrm{La} 2 \rightarrow \mathrm{O})=6.0,{ }^{2} \mathrm{ECoN}(\mathrm{Si} \rightarrow \mathrm{O})=4.0$. Owing to the limited distortion of the polyhedra, ${ }^{n} \mathrm{ECoN}(i j)$ is not much different from ${ }^{1} \mathrm{ECoN}(i j)$ and the $\mathrm{CD}$ method gives relatively satisfactory results, but does not account for the apparent OUB effect. The CD-IT method, through the iterative calculation of the scale factors for polyhedra coordinating both Se 
Table 1

List of the structures refined by single-crystal neutron diffraction containing hydrogen bonds and used to refine the contraction parameter (see $\S 4$ ).

'Code' is the collection code given in the Inorganic Crystal Structure Database (Bergerhoff et al., 1983).

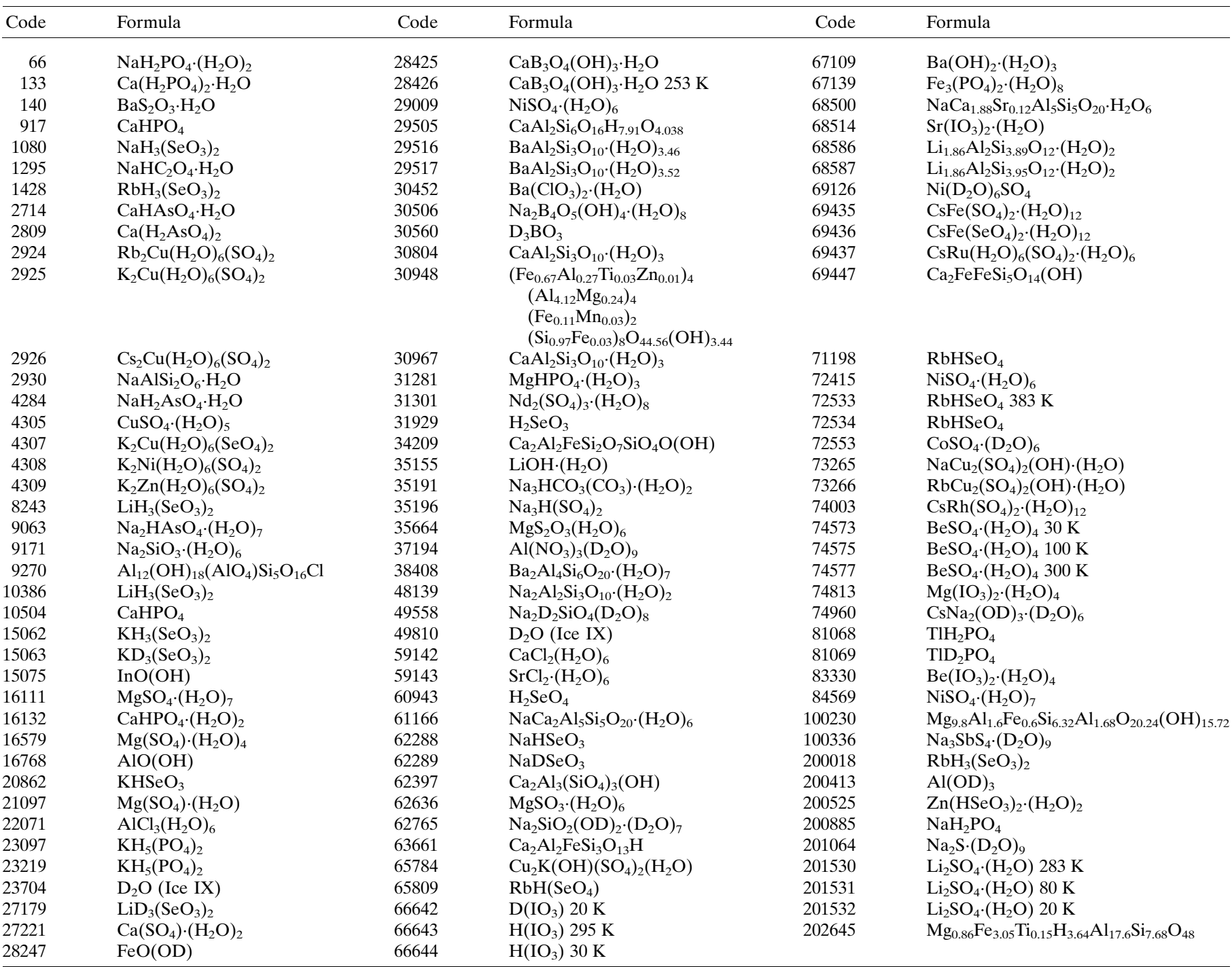

and $\mathrm{O}$ (namely the two La-centred polyhedra), results in a better charge distribution.

\subsection{Analysis of oxides of pentacoordinated cations}

We have chosen oxides containing pentacoordinated cations as a suitable test for the iterative CD (CD-IT) method, because the fivefold coordination polyhedra have often a rather irregular shape. To investigate similarities and dissimilarities related to the cation occupation, we have selected well refined structures in which the fivefold coordination polyhedra are relatively close in shape to either a square pyramid or a trigonal bipyramid, namely the two polyhedra of highest symmetry. Structures in which $\sigma$, as defined in (18), was larger than $10 \%$, or in which the published coordinates did not agree with the bond distances, were excluded. Structures investi- gated by X-ray diffraction and including $\mathrm{H}$ atoms were also excluded, because of the uncertainty that is often associated with their positions, especially when heavy atoms are present. Table 5 lists 74 structures selected on the basis of these criteria.

Fig. 1 (square pyramids) and Fig. 2 (trigonal bipyramids) show the plot $\sum_{s_{\text {(basal) }}} Q(1 s)$ versus $\sum_{s_{\text {(apical) }}} Q(1 s)$ ( $r=1$ because oxygen is the only anion in these compounds). The moderate scatter of point around the fitting straight line shows that the whole valence balance tends to be kept around the pentacoordinated cation. The two most evident deviations are explained as follows.

(i) Triclinic $\alpha-\mathrm{CaSi}_{2} \mathrm{O}_{5}$, space-group type $\bar{P} 1$, is obtained by synthesis at high pressure and high temperature and shows pentacoordinated $\mathrm{Si}$, interpreted both as a distorted trigonal bipyramid (Angel et al., 1996) and as a distorted square pyramid (Kudoh \& Kanzaki, 1998). The deviation of this 
Table 2

$\mathrm{CD}$, CD-IT and BV calculation for $\mathrm{V}_{1.08} \mathrm{P}_{0.92} \mathrm{O}_{5}$ (Jordan \& Calvo, 1976).

BS: Brown \& Shannon (1973); BW: Brown \& Wu (1976); BA: Brown \& Altermatt (1985). $\sigma$ measures the deviation of $Q$ with respect to $q$ and is defined as $\sigma=\left[\Sigma_{i}\left(q_{i}-Q_{i}\right)^{2} /(N-1)\right]^{1 / 2}$. For atoms all with the same $q, \sigma$ reduces to the classical standard uncertainty.

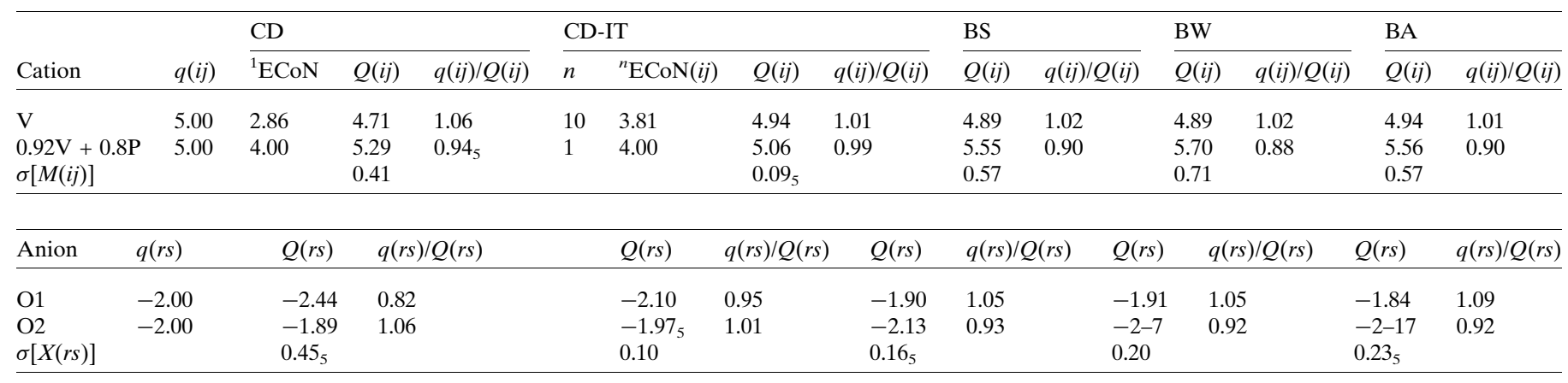

Table 3

CD, CD-IT and BV calculation for hydrogen-bond containing $\mathrm{NaH}_{2} \mathrm{PO}_{4} \cdot \mathrm{H}_{2} \mathrm{O}$ (Bartl et al. 1976).

Symbols as in Table 2 BS-H, BW-H and BA-H indicate calculations in which the $R-s$ curves specific for $\mathrm{H}-\mathrm{O}$ and $\mathrm{Na}-\mathrm{O}$ bonds have been employed (Brown, 1987).

\begin{tabular}{|c|c|c|c|c|c|c|c|c|c|c|c|c|c|c|c|c|c|c|c|}
\hline \multirow[b]{2}{*}{ Cation } & \multirow[b]{2}{*}{$q(i j)$} & \multicolumn{3}{|l|}{$\mathrm{CD}$} & \multicolumn{3}{|l|}{ CD-IT } & \multicolumn{2}{|l|}{ BS } & \multicolumn{2}{|l|}{ BS-H } & \multicolumn{2}{|l|}{ BW } & \multicolumn{2}{|c|}{ BW-H } & \multicolumn{2}{|l|}{ BA } & \multicolumn{2}{|l|}{ BA-H } \\
\hline & & ${ }^{1} \mathrm{ECoN}$ & $Q(i j)$ & $\begin{array}{l}q(i j) / \\
Q(i j)\end{array}$ & ${ }^{n} \mathrm{ECoN}(i j)$ & $Q(i j)$ & $\begin{array}{l}q(i j) / \\
Q(i j)\end{array}$ & $Q(i j)$ & $\begin{array}{l}q(i j) / \\
Q(i j)\end{array}$ & $Q(i j)$ & $\begin{array}{l}q(i j) / \\
Q(i j)\end{array}$ & $Q(i j)$ & $\begin{array}{l}q(i j) / \\
Q(i j)\end{array}$ & $Q(i j)$ & $\begin{array}{l}q(i j) / \\
Q(i j)\end{array}$ & $Q(i j)$ & $\begin{array}{l}q(i j) / \\
Q(i j)\end{array}$ & $Q(i j)$ & $\begin{array}{l}q(i j) / \\
Q(i j)\end{array}$ \\
\hline Na1 & 1.00 & 5.79 & 0.95 & 1.05 & 5.82 & 1.00 & 0.99 & 1.04 & 0.96 & 1.10 & 0.91 & 1.06 & 0.94 & 1.10 & 0.91 & 1.10 & 0.91 & 1.10 & 0.91 \\
\hline H1 & 1.00 & 1.00 & 0.90 & $1.11_{5}$ & 1.83 & $1.03_{5}$ & 0.97 & 0.96 & 1.06 & 1.07 & 0.94 & 0.97 & 1.03 & 1.07 & 0.94 & 0.84 & 1.20 & 1.07 & 0.94 \\
\hline $\mathrm{H} 2$ & 1.00 & 1.00 & 0.91 & $1.09_{5}$ & 1.85 & 1.03 & 0.97 & 0.98 & 1.02 & 1.09 & 0.91 & 0.99 & 1.01 & 1.09 & 0.91 & 0.86 & 1.16 & 1.09 & 0.91 \\
\hline H3 & 1.00 & 1.00 & 0.92 & 1.08 & 1.59 & 0.95 & 1.05 & 0.96 & 1.04 & 1.11 & 0.90 & 0.97 & 1.03 & 1.11 & 0.91 & 0.86 & 1.17 & 1.11 & 0.90 \\
\hline H6 & 1.00 & 1.00 & 0.85 & 1.17 & 1.43 & 0.99 & 1.01 & 0.80 & 1.25 & 0.99 & 1.01 & 0.81 & 1.23 & 0.99 & 1.01 & 0.82 & 1.22 & 0.99 & 1.01 \\
\hline$\sigma[M(i j)]$ & & & 0.28 & & & 0.04 & & 0.11 & & 0.09 & & 0.10 & & 0.08 & & 0.15 & & 0.09 & \\
\hline
\end{tabular}

\begin{tabular}{lccccccccccccccccc}
\hline Anion & $q(r s)$ & $Q(r s)$ & $Q(r s)$ & $Q(r s)$ & $Q(r s)$ & $Q(r s)$ & $Q(r s)$ & $Q(r s)$ & $Q(r s)$ & $Q(r s)$ & $Q(r s)$ & $Q(r s)$ & $Q(r s)$ & $Q(r s)$ & $Q(r s)$ & $Q(r s)$ & $Q(r s)$ \\
\hline O1 & -2.00 & $-1.66_{5}$ & 1.20 & -1.99 & 1.00 & -1.83 & 1.09 & -1.84 & 1.08 & -1.88 & 1.06 & -1.90 & 1.05 & -1.77 & 1.13 & -1.87 & 1.07 \\
O2 & -2.00 & -1.40 & 1.42 & -2.14 & $0.93_{5}$ & -1.98 & 1.01 & -1.94 & 1.03 & -2.03 & 0.99 & -1.98 & 1.01 & -1.66 & 1.21 & -1.96 & 1.02 \\
O3 & -2.00 & -2.23 & 0.90 & -1.91 & 1.05 & -1.98 & 1.01 & -2.10 & 0.95 & -1.98 & 1.01 & -2.09 & 0.96 & -1.95 & 1.03 & -2.07 & 0.97 \\
O4 & -2.00 & -2.19 & 0.91 & -1.86 & 1.04 & -1.96 & 1.02 & -2.08 & 0.96 & -1.96 & 1.02 & -2.06 & 0.97 & -1.92 & 1.04 & -2.05 & 0.98 \\
O5 & -2.00 & $-2.16_{5}$ & 0.92 & -2.10 & 0.95 & -1.77 & 1.13 & -2.16 & 0.93 & -1.79 & 1.11 & -2.16 & 0.93 & -1.81 & 1.10 & -2.16 & 0.93 \\
O6 & -2.00 & -2.35 & 0.85 & -2.01 & 0.99 & -1.93 & 1.04 & -2.32 & 0.86 & -1.96 & 1.02 & -2.32 & 0.86 & -1.99 & 1.01 & -2.32 & 0.86 \\
$\sigma[X(r s)]$ & & 0.375 & & 0.11 & & 0.13 & & 0.18 & & 0.11 & & 0.17 & & 0.21 & & 0.17 & \\
\hline
\end{tabular}

structure from the general trend (white star symbol in both Figs. 1 and 2) supports the instability of the phase as already noted by the quoted authors on the basis of bond strength imbalance around $\mathrm{Ca}$. In fact, the synthesis product always contains some monoclinic $\beta$-CaSi ${ }_{2} \mathrm{O}_{5}$ (titanite-type structure) to which it converts at ambient pressure.

(ii) $\beta-\mathrm{NaVSiO}_{3}$, space-group type Pnma (black star in Fig. 2), is the low-temperature phase of the sodium metavanadate with pentacoordinated V. Metavanadates, also known as 'catena-vanadates' because they are built on infinite chains of corner-sharing tetrahedra as in pyroxenes, are known with a wide range of substitutions in the metal site: $\mathrm{Li}, \mathrm{Na}, \mathrm{K}, \mathrm{Rb}, \mathrm{Cs}$, $\mathrm{Ca}, \mathrm{Ba}, \mathrm{Tl}, \mathrm{Np}$. In their hydrated forms they contain $\mathrm{V}$ in fivefold coordination, either as a square pyramid (more common) or as a trigonal bipyramid (see e.g. Evans, 1960; Björnberg \& Hedman, 1977). Instead, anhydrous metavanadates are usually isostructural with diopside and have $\mathrm{V}$ in tetrahedral coordination. Besides $\beta-\mathrm{NaVSiO}_{3}$, we are aware of only one other anhydrous metavanadate with pentacoordinated V, $\mathrm{CaV}_{2} \mathrm{O}_{6}$ (Perez et al., 1970), which however has one $\mathrm{O}$ atom unrealistically underbonded and a $q(i j) / Q(i j)$ largely deviating from 1 ; for these reasons it is not considered in our survey. The high-temperature phase of $\mathrm{NaVSiO}_{3}$, the $\alpha$ polymorph, with a ferroparaelectric transition at about $653 \mathrm{~K}$ (Ramani et al., 1975), is instead isostructural with diopside. The $\beta$ polymorph has been obtained only through topotactic dehydration of $\mathrm{NaVO}_{3} \cdot 2 \mathrm{H}_{2} \mathrm{O}$, from which the chains of trigonal bipyramids are inherited (Kato \& Takayama, 1984), 
Table 4

CD versus CD-IT calculation for $\mathrm{La}_{2} \mathrm{SeSiO}_{4}$ (Brennan \& Ibers, 1991).

\begin{tabular}{|c|c|c|c|c|c|c|c|c|}
\hline \multirow[b]{2}{*}{ Cation } & \multirow[b]{2}{*}{$q(i j)$} & \multicolumn{3}{|l|}{$\mathrm{CD}$} & \multicolumn{4}{|l|}{ CD-IT } \\
\hline & & ${ }^{1} \mathrm{ECoN}$ & $Q(i j)$ & $q(i j) / Q(i j)$ & $\overline{{ }^{n} \mathrm{ECoN}(i j)}$ & $Q(i j)$ & $q(i j) / Q(i j)$ & Scale factor \\
\hline La1 & 3.00 & 8.46 & 3.00 & 1.00 & 8.54 & 2.99 & 1.00 & La1-Se: 0.33 \\
\hline $\mathrm{La} 2$ & 3.00 & 8.96 & 3.05 & $0.98_{5}$ & 9.09 & 3.01 & 1.00 & La1-O: 0.67 \\
\hline $\mathrm{Si}$ & & 4.00 & 3.95 & 1.01 & 4.00 & 4.00 & 1.00 & La2-Se: 0.33 \\
\hline$\sigma[M(i j)]$ & & & 0.05 & & & 0.01 & & La2-O: 0.67 \\
\hline Anion & $q(r s)$ & & $Q(r s)$ & $q(r s) / Q(r s)$ & & $Q(r s)$ & $q(r s) / Q(r s)$ & $\mathrm{Si}-\mathrm{Se}: 0.00$ \\
\hline $\mathrm{Se}$ & -2.00 & & -1.89 & 1.06 & & -2.00 & 1.00 & $\mathrm{Si}-\mathrm{O}: 1.00$ \\
\hline O1 & -2.00 & & -2.07 & 0.97 & & -2.03 & $0.98_{5}$ & \\
\hline $\mathrm{O} 2$ & -2.00 & & $-1.98_{5}$ & 1.01 & & -1.97 & 1.02 & \\
\hline$\sigma[X(r s)]$ & & & 0.09 & & & 0.03 & & \\
\hline
\end{tabular}

Symbols as in Table 2.

$-z$ around the centre of the cuboctahedron of fluorine and forms only one very long bond with $M^{\prime}$, with almost negligible (0.06) bond weight. Table 6 gives the results of the CD-IT calculation. In the first column, the calculation of the entire structure is shown. The large deviation $q(\mathrm{~F} 6) / Q(\mathrm{~F} 6)$ is expected, because F6 practically does not receive bond weights from any cation. Despite this fact, the distribution on the cations shows that the structure is correct. The second column of Table 6 gives the calculation for the $\left[M_{7} M_{6}^{\prime} \mathrm{F}_{30}\right]^{+}$part of the structure, namely for the structure from which $\mathrm{F}(6)$ has been and irreversibly transforms to the $\alpha$ polymorph upon heating at 676-678 K (Lukács \& Strusievici, 1962; Marumo et al., 1974). $\beta-\mathrm{NaVSiO}_{3}$ thus represents an unstable phase and, as for $\alpha-\mathrm{CaSi}_{2} \mathrm{O}_{5}$, that is reflected by its divergence from the regression line in Fig. 2.

\subsection{Polyionic structures and 'dynamic' structures}

As stated above, polyions are not accounted for by the $M(i j)$ $\rightarrow A(r s)$ bonding patterns assumed by the $\mathrm{CD}$ method, which is thus in principle not applicable to polyionic structures. Here we briefly analyse the case of structures containing anions which are bonded to none of the cations but to complex cations of the type $\left[M X_{n}\right]^{m+}$. One typical example is that of the compounds $M_{7} M_{6}^{\prime} \mathrm{F}_{31}$, such as $\mathrm{Na}_{7} \mathrm{Zr}_{6} \mathrm{~F}_{31}$ (Burns et al., 1968) and $\mathrm{K}_{7} \mathrm{Th}_{6} \mathrm{~F}_{31}$ (Brunton, 1971). The crystallographically independent atoms in these structures are one $M$, one $M^{\prime}$ and six F. $\mathrm{F}(6)$ is statistically distributed between two positions at $+z$ and

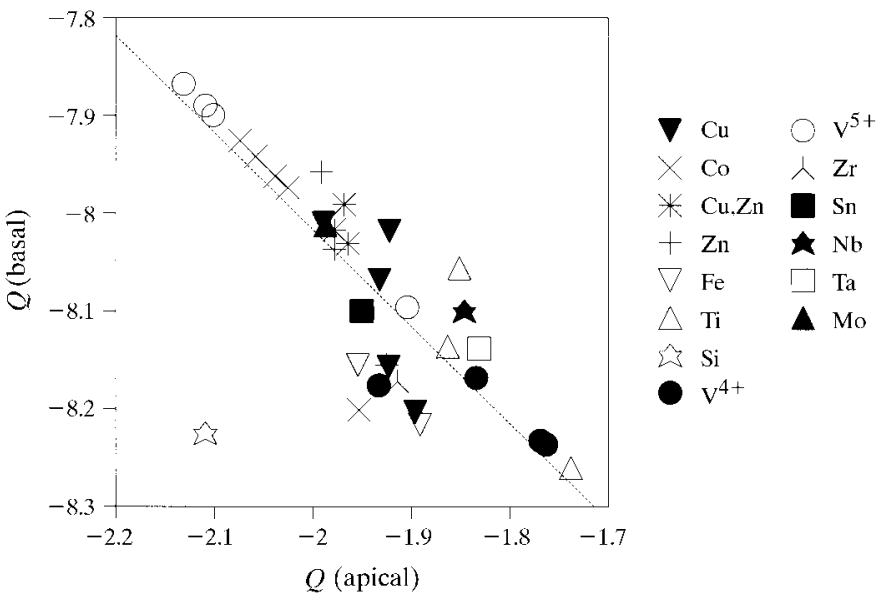

Figure 1

$\sum_{s_{(\text {basal }}} Q(1 s)$ versus $Q(1$,apical) for the polyhedra close to square pyramids from sample 1-35 in Table 5. With the clear exception of $\alpha$ $\mathrm{CaSi}_{2} \mathrm{O}_{5}$ (white star), the data can be roughly interpolated by a regression line. removed. The $q / Q$ on both the cations and the anions is very close to 1 and the slight increase in $\sigma[M(i j)]$ is an artefact from having used a non-neutral structure, for which (14) is not satisfied. Notice that the sign of the charge unbalance is necessarily inverted when $\mathrm{CD}$ is applied to a non-neutral structure: in other words, $-\sum_{r} \sum_{s} q(r s) h(r s)<$ $\sum_{i} \sum_{j} q(i j) h(i j) \quad$ results in $-\sum_{r} \sum_{s} n^{\prime} Q(r s) h(r s)>$ $\sum_{i} \sum_{j} Q(i j) h(i j)$ and vice versa. This sign inversion derives from the fact that $q(i j)$ is used to compute $Q(r s),(9)$, and $q(r s)$ is used to compute $Q(i j)$, (10). When an anion is instead completely isolated from the framework of cations the structure cannot be analysed in terms of the CD method, at least in its present form. For example, the structure of $\mathrm{PBr}_{5}$ (Gabes \& Olie, 1970) consists of tetrahedral $\mathrm{PBr}_{4}^{+}$ions and $\mathrm{Br}^{-}$ions, and is more correctly written as $\left[\mathrm{PBr}_{4}\right]^{+} \mathrm{Br}^{-}$. It contains one crystallographically independent type of $\mathrm{P}$ and four crystallographically independent types of $\mathrm{Br} ; \mathrm{Br}(4)$ is not bonded to $\mathrm{P}$, but is surrounded by four $\mathrm{Br}$ atoms each belonging to a different $\mathrm{PBr}_{4}^{+}$unit, with distances much smaller than the van

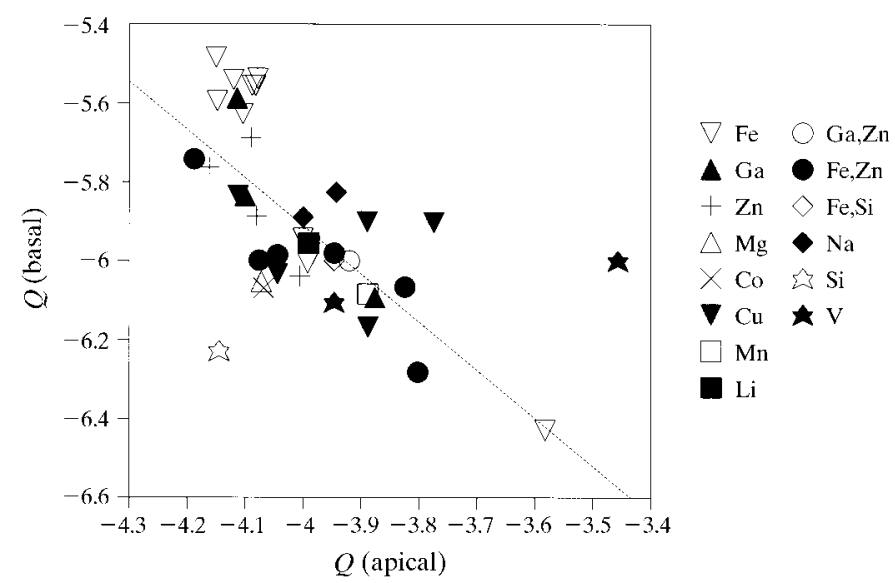

Figure 2

$\sum_{s_{\text {(basal }}} Q(1 s)$ versus $\sum_{s_{\text {(apical }}} Q(1 s)$ for the polyhedra close to trigonal bipyramids from sample $36-74$ in Table 5 . With only two clear exceptions $\left[\alpha-\mathrm{CaSi}_{2} \mathrm{O}_{5}\right.$ (white star) and $\beta-\mathrm{NaVO}_{3}$ (black star)], the data can be roughly interpolated by a regression line. 
Table 5

Structures used to draw the plots in Figs. 1 and 2.

Samples 1-35 have pentacoordinated cations in polyhedra close to square pyramids, samples 36-74 in polyhedra close to trigonal bipyramids. Multiple occurrences of the same sample indicate the presence of more than one pentacoordinated cation. For $\mathrm{YbFeMnO}_{4}$, the $Q(\mathrm{O} 1)$ has been scaled to s.o.f. $=1$.

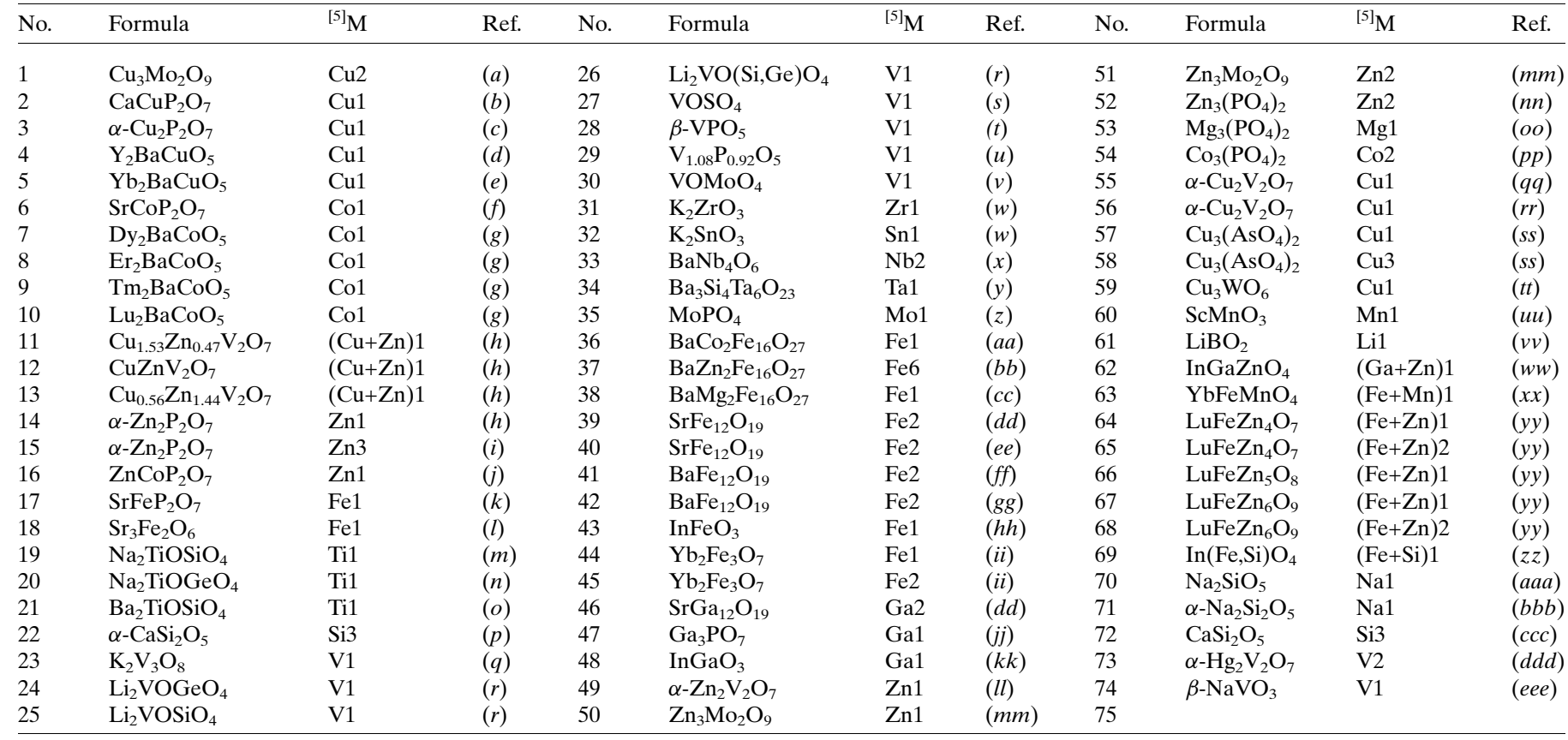

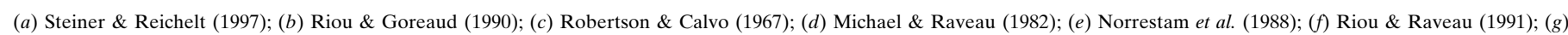

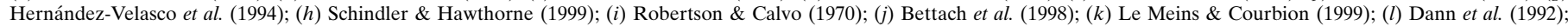

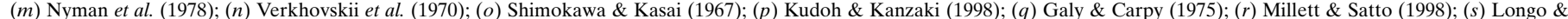

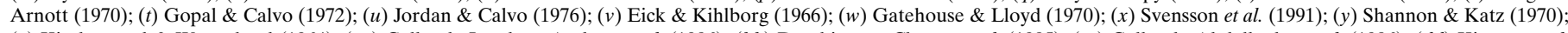

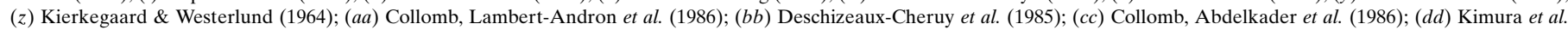

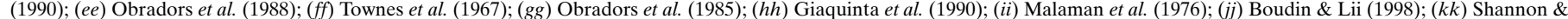

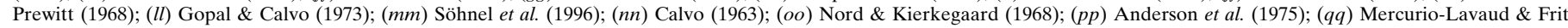

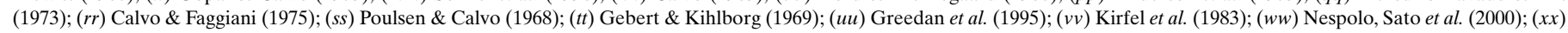

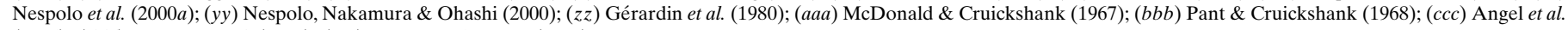
(1996); (ddd) Quarton et al. (1973); (eee) Kato \& Takayama (1984).

der Waals separations. $\Delta q(\mathrm{P} \rightarrow \mathrm{Br} 4)=0$ and thus $Q(\mathrm{Br} 4)=0$. Equation (14) is no longer satisfied and thus (16) is also violated, namely $Q(\mathrm{P}) \neq q(\mathrm{P})$, despite the structure containing only one type of crystallographically independent cation. When dealing with polyionic structures, the limits of the approximation of attributing point charges to the atoms may become critical. The distribution of electron density due to the valence electrons is actually represented by wavefunctions defined in the whole crystal space and the description in terms of point charges is obviously an approximation. However, the Madelung Part of the Lattice Energy, MAPLE (Hoppe, $1970 b$ ), which is also based on a point-charge description of the structure, also takes into account interactions between neighbours bearing 'charges' of the same sign: as a matter of fact, MAPLE for all these halide compounds shows complete agreement with the MAPLE sum of the binary constituents, confirming the correctness of the structures (R. Hoppe, unpublished results).

The CD method is also not directly applicable to 'dynamic' structures, i.e. structures containing disordered (e.g. rotating) ionic groups. A typical example is that of $\mathrm{NF}_{4}^{+} \mathrm{BF}_{4}{ }^{-}$(Christe $e t$ al., 1988). The crystal structure determined by X-ray diffraction represents simply a static model, whereas in the real structure the $\mathrm{BF}_{4}^{-}$group rotates around its centre of gravity and averages the F-F repulsion effects, as shown by vibrational spectra. The atomic positions in the static model do not represent energy minima, but rather a time-average. The $\mathrm{CD}$ calculation gives $Q(\mathrm{~N})=Q(\mathrm{~B})=4.0$, whereas $q(\mathrm{~N})=5.0$ and $q(\mathrm{~B})=3.0$. The large disagreement shows and confirms that the static model is inadequate to describe the structure of this compound.

Dynamic structures may be treated by substituting the complex ion(s) with fictive ion(s), bringing the charge of the complex ion(s) and location at the immobile centre(s). Clearly, the crystal chemical criteria used in normal structures to evaluate the soundness of the structure (first of all bond lengths and bond angles) do not apply and these structures have to be treated case by case.

\section{Discussion}

The concept of 'bond strength' and its development in the form of 'bond valence' derives from the XIX century theory of valence and is based on the idea that the total valence of an atom can be distributed among the bonds in which that atom is involved, so that the 'Valence Sum Rule' is obeyed: the total 
Table 6

CD-IT calculation for $\mathrm{Na}_{6} \mathrm{Zr}_{7} \mathrm{~F}_{31}$ (Burns et al., 1968), with and without taking into account $\mathrm{F} 6$.

Symbols as in Table 2.

\begin{tabular}{|c|c|c|c|c|c|c|c|c|c|}
\hline \multirow[b]{2}{*}{ Cation } & \multirow[b]{2}{*}{$q(i j)$} & \multirow[b]{2}{*}{$h(i j)$} & \multirow[b]{2}{*}{ s.o.f. $(i j)$} & \multicolumn{3}{|l|}{ With F6 } & \multicolumn{3}{|c|}{ Without F6 } \\
\hline & & & & ${ }^{n} \mathrm{ECoN}$ & $Q(i j)$ & $q(i j) / Q(i j)$ & ${ }^{n} \mathrm{ECoN}(i j)$ & $Q(i j)$ & $q(i j) / Q(i j)$ \\
\hline $\mathrm{Na} 1$ & 1.00 & 18 & 1.00 & 7.35 & 0.95 & 1.05 & 7.35 & 0.95 & 1.06 \\
\hline $\mathrm{Na} 2$ & 1.00 & 3 & 1.00 & 7.69 & $0.93_{5}$ & 1.07 & 7.69 & 0.93 & $1.07_{5}$ \\
\hline $\mathrm{Zr}$ & 4.00 & 18 & 1.00 & 7.96 & 4.06 & 0.99 & 7.81 & 3.90 & 1.03 \\
\hline$\sigma[M(i j)]$ & & & & & 0.07 & & & $0.09_{5}$ & \\
\hline$\Sigma_{i} \Sigma_{j} q(i j) h(i j)$ & & & & & 93 & & & 90 & \\
\hline
\end{tabular}

\begin{tabular}{llllllll}
\hline Anion & $q(r s)$ & $h(r s)$ & s.o.f. $(r s)$ & $Q(r s)$ & $q(r s) / Q(r s)$ & $Q(r s)$ & $q(r s) / Q(r s)$ \\
\hline F1 & -1.00 & 18 & 1.00 & -1.03 & 0.97 & -1.03 & 0.97 \\
F2 & -1.00 & 18 & 1.00 & -1.07 & 0.93 & -1.08 & 0.93 \\
F3 & -1.00 & 18 & 1.00 & -1.05 & 0.95 & $-1.05_{5}$ & 0.95 \\
F4 & -1.00 & 18 & 1.00 & -0.95 & 1.06 & -0.95 & 1.05 \\
F5 & -1.00 & 18 & 1.00 & -1.04 & 0.96 & -1.05 & 0.96 \\
F6 & -1.00 & 18 & 0.50 & -0.10 & 5.25 & - & - \\
$\sigma[X(r s)]$ & & & & 0.19 & & 0.06 & \\
$\Sigma_{r} \Sigma_{s} q(r s) h(r s)$ & & & & -93 & & -93 & \\
\hline
\end{tabular}

isomorphous structures (see the example of pyroxenes in Nespolo et al., 1999) and even among similar polyhedra in otherwise different structures, as shown by the example of pentacoordinated cations in the previous section. When, however, the distortion increases and/or the range of bond lengths around a given site widens, cation-anion specific curves, and not only cation-anion specific parameters, are required (Brown, 1987). The CD method, instead, in its iterative extension presented here, does not require any modification, with the only exception of a different contraction parameter for the case of hydrogen bonds.

One aspect that the CD method inherits from the old valence

bond valence received by each atom is equal to its atomic valence. The atomic valence is computed as the sum of bond valences obtained using each bond length as the independent variable in the empirical $R-s$ curves, whose parameters specifically depend on the cation-anion pair (Brown, 1977, 1992). The bond valence is interpreted as the number of electrons pairs associated with a bond, which practically indicates the electron density along that bond. Obviously, only a rather rough measure of electron density can be expected by using the bond distances in an empirical curve, especially in the presence of electronic or steric distortions.

The CD method fundamentally differs from other methods that investigate the connectivity of a crystal structure in being essentially a geometric analysis of the coordination polyhedra centred on each cation site, which exploits the observed distances only instead of comparing them with subjective 'standard values'. The bond weight, (5), is a purely geometric concept which quantitatively tells how much the anion $A(r s)$ at one extreme of the bond $d(i j \rightarrow r s)_{L}$ is attracted by the cation $M(i j)$, in terms of the contribution of that bond to ECoN. The bond weight is not directly related to the electron density of the bond. In fact, the charge - corresponding to the atomic valence in the BV language - is distributed and not computed among each bond, see (8). The equivalent of the Valence Sum Rule strictly applies to $Q(i j)$, but not to $Q(r s)$. This is reflected on the different roles played by the two charges: the measure of the OUB effects by $Q(r s)$ and evaluation of the applicability, including structure reliability, by $Q(i j)$.

Structures with coordination polyhedra of limited distortion and with bonds restricted to within a small range of lengths can be analysed satisfactorily in terms of bond valences, resonance bond numbers or bond weights, but the kind of information conveyed by these methods is basically different. The advantages of the $\mathrm{CD}$ method reside in its independence from the chemical composition, temperature, pressure and in its suitability to reveal similarities and dissimilarities among theory is in assuming the cations as the centre of the coordination polyhedra. Although this description is suitable for most compounds, examples have been reported of complexes having anion-centred groups which are condensed in a more complicated manner than the cation-centred groups (e.g. Krivovichev et al., 1997, 1998). The CD equations can be rewritten in terms of anion-centred polyhedra. In cases where one or more anions belong only to the external shell of coordination of any cations, an anion-centre description may give better results (R. Hoppe, unpublished results).

Mackay \& Finney (1973) have pointed out that the valencesum rule is equivalent to Kirchhoff's current law and Rutherford (1990) has analysed the bond-valence network in terms of graph theory. These insights in the valence-sum rule opened new frontiers in predicting bond lengths by using theoretical values of the bond valences. Rutherford (1998) has demonstrated that the RBN method is a better predictor than the BV method in most cases, but the BV method has the advantage of a much simpler computation. Since the CD method is practically independent from the chemical compound, it is less directly applicable to the prediction of bond lengths. However, its capability of analysing the reliability of a structure can be exploited to evaluate the results of the prediction according to $\mathrm{BV}$ or $\mathrm{RBN}$, and eventually indicate in which direction the results of that prediction need to be modified.

This research has been developed during a post-doctoral program ('Domestic Research Fellowship', MN) supported by the Japan Science and Technology Corporation. We are grateful to Professor Mario Nardelli (University of Parma) for his constructive criticism. MN expresses his gratitude to the staff of the Institut für Anorganische und Allgemeine Chemie of the Justus-Liebig Universität, and particularly to Dr Michael Serafin, for their kindness during his visit in Giessen 
(October 2000). GF acknowledges Italian National Research Council (CNR) and Ministry of University and of Technology and Scientific Research (MURST: 'Transformation, reactions, ordering in minerals' $40 \%$ project) for financial support.

\section{References}

Anderson, J. B., Kostiner, E., Miller, M. C. \& Rea, J. R. (1975). J. Solid State Chem. 14, 372-377.

Angel, R. J., Ross, N. L., Seifert, F. \& Fliervoet, T. F. (1996). Nature, 384, 441-444.

Balić Žunić, T. \& Makovicky, E. (1996). Acta Cryst. B52, 78-81.

Bartl, H. (1970). Neues Jahrb. Mineral. Monatsh. pp. 552-557.

Bartl, H., Catti, M. \& Ferraris, G. (1976). Acta Cryst. B32, 987-994.

Bergerhoff, G., Hundt, R., Sieves, R. \& Brown, I. D. (1983). J. Chem. Inf. Comput. Sci. 23, 66-69.

Bettach, M., Benkhouja, K., Zahir, M., Rissouli, K., Sadel, A., Giorgi, M. \& Pierrot, M. (1998). Acta Cryst. C54, 1059-1062.

Björnberg, A. \& Hedman, B. (1977). Acta Chem. Scand. A, 31, 579584 .

Boisen, M. B., Gibbs, G. V. \& Zhang, Z. G. (1988). Phys. Chem. Miner. 15, 409-415.

Boudin, S. \& Lii, K.-H. (1998). Acta Cryst. C54, 5-7.

Brennan, Th. D. \& Ibers J. A. (1991). Acta Cryst. C47, 1062-1064.

Brown, I. D. (1977). Acta Cryst. B33, 1305-1310.

Brown, I. D. (1987). Phys. Chem. Mineral. 15, 30-34.

Brown, I. D. (1992). Acta Cryst. B48, 553-572.

Brown, I. D. \& Altermatt, D. (1985). Acta Cryst. B41, 244-247.

Brown, I. D. \& Shannon, R. D. (1973). Acta Cryst. A29, 266-282.

Brown, I. D. \& Wu, K. K. (1976). Acta Cryst. B32, 1957-1959.

Brunton, G. (1971). Acta Cryst. B27, 2290-2292.

Burns, J. H., Ellison, R. D. \& Levy, H. A. (1968). Acta Cryst. B24, 230237.

Calvo, C. (1963). J. Phys. Chem. Solids, 24, 141-149.

Calvo, C. \& Faggiani, R. (1975). Acta Cryst. B31, 603-605.

Chiari, G. (1990). Acta Cryst. B46, 717-723.

Chiari, G. \& Ferraris, G. (1982). Acta Cryst. B38, 2331-2341.

Collomb, A., Abdelkader, O., Wolfers, A., Guitel, J. C. \& Samaras, D. (1986). J. Magn. Magn. Mater. 58, 247-253.

Collomb, A., Lambert-Andron, B., Boucherle, J. X. \& Samaras, D. (1986). Phys. Status Solidus A, 96, 385-395.

Christe, K. O., Lind, M. D., Thorup, N., Russell, D. R., Fawcett, J. \& Bau, R. (1988). Inorg. Chem. 27, 2450-2454.

Dann, S. E., Weller, M. T. \& Currie, D. B. (1992). J. Solid State Chem. 97, 179-185.

Deschizeaux-Cheruy, M. N., Vallet-Regi, M. \& Joubert, J. C. (1985). J. Solid State Chem. 57, 234-239.

Eick, H. A. \& Kihlborg, L. (1966). Acta Chem. Scand. 20, 722-729.

Evans, H. T. Jr (1960). Z. Kristallogr. 114, 257-277.

Ferraris, G., Gula, A., Ivaldi, G., Nespolo, M., Sokolova, E., Uvarova,

Y. \& Khomyakov, A. P. (2001). Eur. J. Mineral. In the press.

Ferraris, G. \& Ivaldi, G. (1988). Acta Cryst. B44, 341-344.

Gabes, W. \& Olie, K. (1970). Acta Cryst. B26, 443-444.

Galy, J. \& Carpy, A. (1975). Acta Cryst. B31, 1794-1795.

Gatehouse, B. M. \& Lloyd, D. J. (1970). J. Solid State Chem. 2, 410415.

Gebert, E. \& Kihlborg, L. (1969). Acta Chem. Scand. 22, 221-231.

Gérardin, R., Alebouyeh, A., Jeannot, F., Courtois, A., Malaman, B. \& Evrard, O. (1980). Mater. Res. Bull. 15, 647-655.

Giaquinta, D. M., Davis, W. M. \& zur Loye, H.-C. (1990). Acta Cryst. C50, 5-7.

Gibbs, G. V., Hill, F. C., Boisen, M. B. \& Downs, R. T. (1998). Phys. Chem. Miner. 25, 585-590.

Gopal, R. \& Calvo, C. (1972). J. Solid State Chem. 5, 432-435.

Gopal, R. \& Calvo, C. (1973). Can. J. Chem. 51, 1004-1009.

Greedan, J. E., Bieringer, M., Britten, J. F., Giaquinta, D. M. \& zur Loye, H.-C. (1995). J. Solid State Chem. 116, 118-130.
Hernández-Velasco, J., Salinas-Sánchez, A., Fernández, F. \& SáezPuche, R. (1994). J. Alloys Compd, 203, 15-22.

Hoppe, R. (1970a). Angew. Chem. Int. Ed. Engl. 9, 25-34.

Hoppe, R. (1970b). Adv. Fluorine Chem. 6, 387-438.

Hoppe, R. (1979). Z. Kristallogr. 150, 23-52.

Hoppe, R., Voigt, S., Glaum, H., Kissel, J., Müller, H. P. \& Bernet, K. (1989). J. Less-Common Met. 156, 105-122.

Jordan, B. D. \& Calvo, C. (1976). Acta Cryst. B32, 2899-2900.

Kato, K. \& Takayama, E. (1984). Acta Cryst. B40, 102-105.

Kierkegaard, P. \& Westerlund, M. (1964). Acta Chem. Scand. 18, 2217-2225.

Kimura, K., Ohgaki, M., Tanaka, K., Morikawa, H. \& Marumo, F. (1990). J. Solid State Chem. 87, 186-194.

Kirfel, A., Will, G. \& Stuart, R. F. (1983). Acta Cryst. B39, 175-185. Krivovichev, S. V., Filatov, S. K. \& Semenova, T. F. (1997). Z. Kristallogr. 212, 411-417.

Krivovichev, S. V., Filatov, S. K. \& Semenova, T. F. (1998). Russ. Chem. Rev. 67, 137-155.

Kudoh, Y. \& Kanzaki, M. (1998). Phys. Chem. Miner. 25, 429-433.

Le Meins, J. M. \& Courbion, G. (1999). Acta Cryst. C55, 481-483.

Longo, J. M. \& Arnott, R. J. (1970). J. Solid State Chem. 1, 394-398.

Lukács, I. \& Strusievici, C. (1962). Z. Anorg. Allg. Chem. 315, 323 326.

McDonald, W. S. \& Cruickshank, D. W. J. (1967). Acta Cryst. 22, 37 47.

Mackay, A. L. \& Finney, J. L. (1973). J. Appl. Cryst. 6, 284-289.

Makovicky, E. \& Balić Žunić, T. (1998). Acta Cryst. B54, 766-773.

Malaman, B., Evrard, O., Tannièeres, N., Courtois, A. \& Protas, J. (1976). Acta Cryst. B32, 749-752.

Marumo, F., Isobe, M., Iwai, S. \& Kondoh, Y. (1974). Acta Cryst. B30, $1628-1630$.

Mercurio-Lavaud, D. \& Frit, B. (1973). Acta Cryst. B29, 2737-2741.

Michael, C. \& Raveau, B. (1982). J. Solid State Chem. 43, 73-80.

Millett, P. \& Satto, C. (1998). Mater. Res. Bull. 33, 1339-1345.

Mohri, F. (2000). Acta Cryst. B56, 626-638.

Naskar, J. P., Hati, S. \& Datta, D. (1997). Acta Cryst. B53, 885-894.

Nardelli, M. (1999). J. Appl. Cryst. 32, 563-571.

Nespolo, M., Ferraris, G. \& Hoppe, R. (2001). J. Ceram. Process. Res. 2, 38-44.

Nespolo, M., Ferraris, G. \& Ohashi, H. (1999). Acta Cryst. B55, $902-$ 916.

Nespolo, M., Isobe, M., Iida, J. \& Kimizuka, N. (2000a). Acta Cryst. B56, 805-810.

Nespolo, M., Isobe, M., Iida, J. \& Kimizuka, N. (2000b). J. Alloys Compd, 313, 59-64.

Nespolo, M., Nakamura, M. \& Ohashi, H. (2000). J. Solid State Chem. 150, 96-103.

Nespolo, M., Sato, A., Osawa, T. \& Ohashi, H. (2000). Cryst. Res. Technol. 35, 151-165.

Nord, A. G. \& Kierkegaard, P. (1968). Acta Chem. Scand. 22, 1466 1474.

Norrestam, R., Hjorth, M. \& Bovin, J. O. (1988). Z. Kristallogr. 183, 245-251.

Nyman, H., O'Keefe, M. \& Bovin, J.-O. (1978). Acta Cryst. B34, $905-$ 906.

Obradors, X., Collomb, A., Pernet, M., Samaras, D. \& Joubert, J. C. (1985). J. Solid State Chem. 56, 171-181.

Obradors, X., Solans, X., Collomb, A., Samaras, D., Rodriguez, J., Pernet, M. \& Font-Altaba, M. (1988). J. Solid State Chem. 72, 218 224.

Pant, A. K. \& Cruickshank, D. W. J. (1968). Acta Cryst. B24, 13-19.

Parthé, E. (1996). Elements of Inorganic Structural Chemistry. PetitLancy: K. Sutter Parthé.

Pauling, L. (1929). J. Am. Chem. Soc. 51, 1010-1026.

Perez, G., Frit, B., Bouloux, J. C. \& Galy, J. (1970). Comp. R. Acad. Sci. Paris Ser. C, 270, 952-953.

Poulsen, S. J. \& Calvo, C. (1968). Can. J. Chem. 46, 917-927. 
Quarton, M., Angenault, J. \& Rimsky, A. (1973). Acta Cryst. B29, 567-573.

Ramani, K., Shaikh, M., Swaminatha, R. \& Viswamitra, M. A. (1975). Ferroelectrics, 9, 49-56.

Riou, D. \& Goreaud, M. (1990). Acta Cryst. C46, 1191-1193.

Riou, D. \& Raveau, B. (1991). Acta Cryst. C47, 1708-1709.

Robertson, B. E. \& Calvo, C. (1967). Acta Cryst. 22, 665-672.

Robertson, B. E. \& Calvo, C. (1970). J. Solid State Chem. 1, 120-133.

Rutherford, J. S. (1990). Acta Cryst. B46, 289-292.

Rutherford, J. S. (1991). Trans. Am. Cryst. Soc. 27, 315-321.

Rutherford, J. S. (1998). Acta Cryst. B54, 204-210.

Schindler, M. \& Hawthorne, F. (1999). J. Solid State Chem. 146, 271276.

Shannon, J. \& Katz, L. (1970). J. Solid State Chem. 1, 399-408.
Shannon, R. D. \& Prewitt, C. T. (1968). J. Inorg. Nucl. Chem. 30, 1389-1398.

Shimokawa, K. \& Kasai, Z. (1967). Science, 156, 1361-1363.

Söhnel, T., Reichelt, W., Oppermann, A., Mattausch, Hj. \& Simopn, A. (1996). Z. Anorg. Allg. Chem. 622, 1274-1280.

Steiner, U. \& Reichelt, W. (1997). Acta Cryst. C53, 1371-1373.

Svensson, G., Kohler, J. \& Simon, A. (1991). J. Alloys Compd, 176, 123-132.

Townes, W. D., Fang, J. H. \& Perrotta, A. J. (1967). Z. Kristallogr. 125, 437-449.

Urusov, V. S. (1995). Acta Cryst. B51, 641-649.

Verkhovskii, Yu. A., Myrashova, E. V. \& Trunov, V. K. (1970). Sov. Phys. Crystallogr. 15, 7-8.

Wang, X. \& Liebau, F. (1996a). Acta Cryst. B52, 7-15.

Wang, X. \& Liebau, F. (1996b). Z. Kristallogr. 211, 437-439. 